\title{
OPTIMAL TRAJECTORIES TOWARDS NEAR-EARTH-OBJECTS USING SOLAR ELECTRIC PROPULSION (SEP) AND GRAVITY ASSISTED MANEUVER
}

\author{
Denilson Paulo Souza dos Santos ${ }^{1}$, Lorenzo Casalino ${ }^{2}$, Guido Colasurdo ${ }^{3}$ \\ Antônio Fernando Bertachini de Almeida Prado ${ }^{4}$ \\ ${ }^{1,4}$ Division of Space Mechanics and Control - INPE \\ C.P. 515, 12227-310 São José dos Campos - SP, Brasil \\ denilson@dem.inpe.br;prado@dem.inpe.br \\ ${ }^{2,3}$ Politecnico di Torino, Corso Duca degli Abruzzi, 24, 10129 Torino, Italy \\ lorenzo.casalino@polito.it; guido.colasurdo@uniromal.it
}

\begin{abstract}
The solar electric propulsion could be the best option for the transports of the future due to its high specific impulse when compared to the chemical propulsion. Electric propellants are being extensively used to assist the propulsion of terrestrial satellites for the maneuvers of orbit correction and as primary propulsion in missions toward other bodies of the solar system.

In this work the optimization of interplanetary missions using solar electric propulsion (SEP) and Gravity Assisted Maneuver to reduce the costs of the mission, is considered. The high specific impulse of electric propulsion makes a Gravity Assisted Maneuver 1 year after departure convenient. Missions for several Near Earth Asteroids will be considered. The analysis suggests criteria for the definition of initial solutions demanded for the process of optimization of trajectories.

Trajectories to the asteroid 2002TC70 are analyzed. Direct trajectories, trajectories with 1 gravity assisted at the Earth and with 2 gravity assisted with the Earth and either Mars are presented. Shall be analyzed missions with thrusters PPS1350 and the Phall 1 for performance comparison. An indirect optimization method will be used in the simulations.
\end{abstract}

Keywords: Astrodynamics, Celestial Mechanics, Space Trajectories.

\section{INTRODUCTION}

The future interplanetary missions will probably use the conventional chemical rockets to leave the sphere of influence of the Earth, and solar electric propulsion (SEP) to accomplish the other maneuvers of the mission.

Both NASA and ESA have launched spacecrafts which used SEP (Solar Electric Propulsion) as the primary propulsion system; NASA's DS1 and ESA's Smart-1 to the moon to comet Borrelly. Indirect optimization methods are suitable for the low thrust trajectories that are used in simulations. A finite force is applied during a finite interval of time and it is necessary to integrate the state equation along the time to know its effect. Several results exist in the literature, starting with the works of Tsien (1953) and Lawden (1955). Other results and references can be found in Prado (1989), Prado and Rios-Neto (1993), Casalino and Colasurdo [1], Santos [2]. The most used method in this model is the so called "primer-vector theory", developed by Lawden (1953 and 1954) $[4,5]$, according to Prado [6, 7], Santos [8, 9]. In this paper, theory of optimal control is applied and a procedure based on the Newton's Method to decide the boundary problems is developed. The Pontryagin's Maximum Principle (PMP) is used to maximize the Hamiltonian associated to the problem and evaluates the optimal structure of the "switching function".

The spacecraft leaves the Earth's sphere of influence with a hyperbolic velocity whose optimal magnitude and the direction will be supplied by the optimization procedure. The initial mass is directly related to the magnitude of the hyperbolic velocity, assuming that a chemical thruster is used to leave a low Earth orbit (LEO). Out of the Earth's sphere of influence, the electric 
propellants is activate and the available power is proportional to the square of the distance from the Sun; the propulsion is provided by one or two "PPS 1350 ion thrusters and Phall1 thrusters (UNB)".

\section{DESCRIPTION OF THE PROBLEM}

The spacecraft will be considered a point with variable mass $\mathrm{m}$ and the trajectory will be analyzed using the patched-conics approach. The time required by the spacecraft to leave the Earth's sphere of influence is neglected and, in this formulation, only equations of motion in the heliocentric reference system will be considered. The spacecraft is influenced by the Sun gravitational acceleration $\vec{g}(r)$ and the propulsion system that the vehicle implements, with a level of thrust $T$. With this formulation, a maneuver including an Earth's flyby can be used to gain energy and velocity, which provokes a discontinuity in the relative state variables of the velocity.

The variables are normalized using the radius of the Earth's orbit, the corresponding circular velocity, and the mass of the spacecraft in stationary orbit as values of reference.

The solar electric Propulsion system will be considered, therefore, the available power and thrust varies with the square of the distance from the sun.

In this problem, the thrust is the only control during the heliocentric arcs, and it will be optimized to get the minimum consumption, that is measured by the final mass of the spacecraft. Since the thrust appears linearly in the equation of motion, a bang-bang control, which consists of alternating ballistic arcs with arcs of maximum thrust, will be required. The trajectory is composed by a succession of ballistic arcs (zerothrust) and arcs of maximum thrust, where the optimal direction will be supplied by the optimization procedure.

The boundary conditions are imposed in a satisfactory way at the junctions between trajectory arcs.

The integration initiates when the spacecraft leaves the Earth's sphere of influence, at the position $\vec{r}_{i}=\vec{r}_{\oplus}\left(t_{i}\right)$ that coincides with the Earth's position, considering the velocity $\vec{v}_{i}$ free. The hyperbolic velocity is given by $\vec{v}_{\infty i}=\vec{v}_{i}-\vec{v}_{\oplus}\left(t_{i}\right)$, assuming that a rocket thruster is used to take the spacecraft from the Low Earth Orbit (LEO) with an impulsive maneuver and that the vehicle mass on LEO is specified. The increment of velocity $(\Delta \mathrm{V})$ demanded to provide the hyperbolic velocity is $\Delta V=\sqrt{v_{\infty i}^{2}+v_{e}^{2}}-v_{c}$, where $\mathrm{v}_{\mathrm{e}}$ and $\mathrm{v}_{\mathrm{c}}$ are the escape and circular velocity at the LEO radius [2]. The initial mass at the exit from the Earth's sphere of influence is,

$$
m_{i}=a-b V_{\infty}-c V_{\infty}^{2}
$$

where,

$\varepsilon\left(1-m_{i}\right)$ is the jettisoned mass of the exhausted motor, which is proportional to the propellant mass. The spacecraft intercepts the Earth and accomplishes Gravity Assisted Maneuvers (Santos et al., 2005) [7 - 9]. The position of the vehicle $\vec{r}_{ \pm}=\vec{r}_{\oplus}\left(t_{ \pm}\right)$is constrained and the magnitude of the hyperbolic excess velocity $\vec{v}_{\infty \pm}=\vec{v}_{ \pm}-\vec{v}_{\oplus}\left(t_{ \pm}\right)$ is continuous $v_{\infty+}^{2}=v_{\infty-}^{2}$ [2].

If the minimum height constraint on the flyby is requested, a condition on the velocity turn angle is added:

$$
\vec{v}_{\infty++}^{T} \vec{v}_{\infty-}=-\cos (2 \phi) v_{\infty-}^{2}
$$

where,

$$
\cos (\phi)=\frac{v_{p}^{2}}{\left(v_{\infty-}^{2}+v_{p}^{2}\right)}
$$

$v_{p}$ is the circular velocity at the low distances allowed for a planet.

$$
\vec{v}_{\infty \pm}=\vec{v}_{i \pm}-\vec{v}_{4}
$$

At the final point (subscript $f$ ), the position and velocity vectors of the spacecraft and the asteroid coincide,

$$
\begin{aligned}
& r_{f}=r_{A}\left(t_{f}\right) \\
& v_{f}=v_{A}\left(t_{f}\right)
\end{aligned}
$$

The theory of optimal control provides the control law and the necessary boundary conditions for optimality. 


\section{OPTIMIZATION PROCEDURES}

The objective is to use the theory of optimal control to maximize the spacecraft final mass.

Dynamical equations are,

$$
\begin{aligned}
& \dot{\vec{r}}=\vec{v} \\
& \dot{\vec{v}}=\vec{g}(\vec{r})+\frac{\vec{T}}{m} \\
& \dot{m}=-\frac{\vec{T}}{c}
\end{aligned}
$$

Applying the theory of optimal control, the Hamiltonian function is defined as (Lawden, 1954) $[3,2]$ :

$$
H=\vec{\lambda}_{r}^{t} \vec{v}+\vec{\lambda}_{v}^{t}\left(\vec{g}+\frac{\vec{T}}{m}\right)-\lambda_{m} \frac{\vec{T}}{c}
$$

An indirect optimization procedure is used to maximize the payload. According to Pontryagin's Maximum Principle the optimal controls maximize $\mathrm{H}$.

The nominal thrust $\mathrm{T}_{\mathrm{o}}$ at $1 \mathrm{AU}$, and the electrical power are (Casalino [2], Santos [9]),

$$
\begin{gathered}
P_{0}=\frac{T_{0} c}{2 \eta} \\
T_{\text {Max }}=\frac{T_{0}}{r^{2}}
\end{gathered}
$$

Optimal control theory provides differential equation for the adjoint equations of the problem (Euler-Lagrange).

Adjoint equations are,

$$
\begin{aligned}
& \dot{\vec{\lambda}}_{r}^{t}=\vec{\lambda}_{v}^{t} \frac{\partial \vec{g}}{\partial \vec{r}}-S_{f} \frac{\partial T}{\partial \vec{r}} \\
& \dot{\vec{\lambda}}_{v}^{t}=-\vec{\lambda}_{r}^{t} \\
& \dot{\vec{\lambda}}_{r}=\lambda_{v}^{t} \frac{\vec{T}}{m^{2}}
\end{aligned}
$$

where, $G=\frac{\partial \vec{g}}{\partial \vec{r}}$.

Optimal control: thrust direction and magnitude are,

$$
\begin{aligned}
& \vec{T} / / \vec{\lambda}_{v} \\
& H=\vec{\lambda}_{r}^{t} \vec{v}+\vec{\lambda}_{v}^{t} G+\vec{T}\left(\frac{\lambda_{v}}{m}-\frac{\lambda_{m}}{c^{\prime}}\right) \\
& S_{f}=\frac{\lambda_{v}}{m}-\frac{\lambda_{m}}{c^{\prime}}
\end{aligned}
$$

where,

$c^{\prime}$ - is the effective exhaust velocity of the rocket thruster;

$$
T_{\text {Max }}=\left\{\begin{array}{l}
\frac{T_{0}}{r^{2}} \rightarrow S_{f}>0 \\
0 \rightarrow S_{f}<0
\end{array}\right.
$$

The necessary optimal conditions $[5,2]$ are:

$$
\begin{aligned}
& \left(H_{j_{-}}+\frac{\partial \varphi}{\partial t_{j_{-}}}+\mu^{t} \frac{\partial \vec{\chi}}{\partial t_{j_{-}}}\right) \delta t_{j_{-}}=0 \\
& \left(H_{j_{+}}-\frac{\partial \varphi}{\partial t_{j_{+}}}-\mu^{t} \frac{\partial \vec{\chi}}{\partial t_{j_{+}}}\right) \delta t_{j_{+}}=0 \\
& \left(\lambda_{j_{-}}^{t}-\frac{\partial \varphi}{\partial \vec{x}_{j_{-}}}-\mu^{t} \frac{\partial \vec{\chi}}{\partial \vec{x}_{j_{-}}}\right) \delta \vec{x}_{j_{-}}=0 \\
& \left(\lambda_{j_{+}}^{t}+\frac{\partial \varphi}{\partial \vec{x}_{j_{+}}}+\mu^{t} \frac{\partial \vec{\chi}}{\partial \vec{x}_{j_{+}}}\right) \delta \vec{x}_{j_{+}}=0
\end{aligned}
$$

Where:

$\vec{\chi}$ : the vector collecting the constraining boundary conditions (see eq. 15 - 18)

$\varphi=\mathrm{m}_{\mathrm{f}}$

At the initial point:

1. $\vec{r}_{0}=\vec{r}_{\oplus} ;$

2. $m_{o}=1-b V_{\infty}-c V_{\infty}^{2}$

3. $\left(\vec{v}_{0}-\vec{v}_{\oplus}\right)^{2}=\vec{v}_{\infty 0}^{2}$;

4. Equations 16 and 18 provide optimal control with $\lambda_{\text {ro }}$ and $\mathrm{T}_{\text {ro }}$ free;

5. the necessary condition optimal of the state is $\vec{\lambda}_{v 0}$ (primer vector) be parallel to the hyperbolic velocity;

At flyby [2]:

1. the equations (15 and 16) are used to obtain the transversality conditions, that implicates in determining the arc time used;

2. in the equations (17 and 18) $\vec{\lambda}_{v i}$ is parallel to the hyperbolic velocity, before and after the 
free flyby maneuver and the magnitude is continuous;

3. the states of Hamiltonian remain continuous through the flyby maneuvers;

4. when the minimum height constraint of the flyby is requested, a condition on the velocity turn angle is added (Eq. 2 and 3).

At the final point:

1. $\vec{\lambda}_{v f}$ is parallel to the hyperbolic velocity, $\vec{\lambda}_{r f}$ is parallel to the radius and $\vec{\lambda}_{r f}^{t} \vec{v}_{f}+\vec{\lambda}_{v f}^{t} \vec{g}=0$;

2. the final values of $\vec{\lambda}_{m f}$ and $H_{f}$ depends on the control model that was considered in the maneuver;

3. the adjoint variable $\vec{\lambda}_{v}$ is zero during the whole trajectory.

\section{MISSION ASTEROID $2002 \mathrm{TC70}$}

\subsection{NUMERICAL ANALYSIS WITH PPS1350 (ESA)}

The characteristics of the spacecraft propulsion system that have been assumed are [11]:

1. the mass of the spacecraft with an altitude of $200 \mathrm{~km}$ in circular LEO is $2133.3 \mathrm{Kg}$;

2. specific impulse $I_{s}=1550 \mathrm{~s}$ :

3. specific energy $\varepsilon=0.06$;

4. $T=2 \cdot 70 \mathrm{mN}$ (thruster PPS 1350 used for the SMART-1 mission to the moon);

5. nominal thruster $\mathrm{T}_{0}=1 \mathrm{UA}$;

6. The time: time $=0$ corresponds to the date 01/01/2000.

The necessary optimal condition were formulated in agreement with the problem; the bang-bang control was used in the formularization with limited power and constraint in the time of flight.

\begin{tabular}{|c|c|}
\hline Name & 2002TC70 \\
\hline Epoch & 54200 \\
\hline$a$ & 1.369831 \\
\hline$e$ & 0.19691574 \\
\hline$i$ & 2.13932 \\
\hline$\Omega$ & 161.89427 \\
\hline$\omega$ & 134.84892 \\
\hline$M$ & 351.6336031 \\
\hline$r_{a}$ & 1.10009 \\
\hline$r_{p}$ & 1.639572 \\
\hline
\end{tabular}

Table 1 - Keplerian Elements

\subsubsection{Simulation without flyby}

Using the optimization procedure we can find optimal trajectories, with the maximization of the spacecraft final mass (i.é., minimum fuel consumption). These trajectories depend on the mission objectives, for example, the performance depends on the mission time length. It is possible to reduce the time with some more spend of propellant.

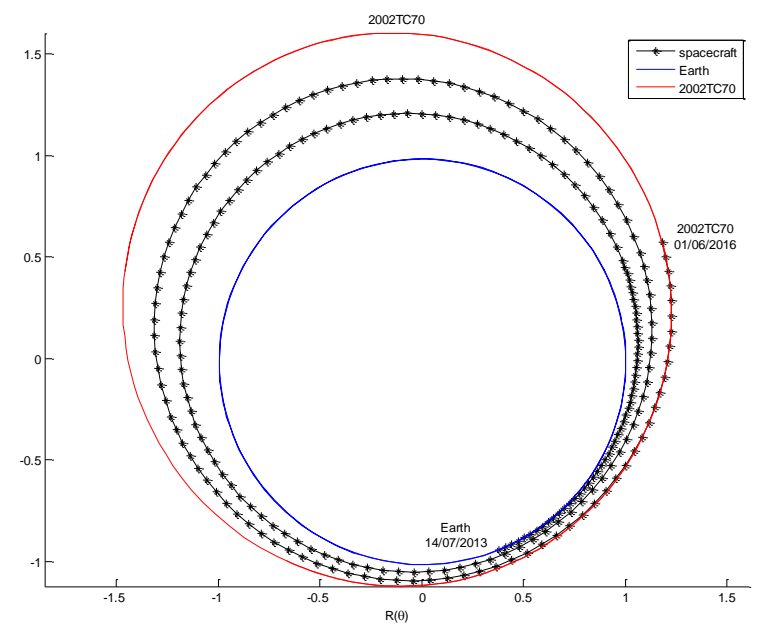

Figure 1 - Trajectory direct (without flyby) to the asteroid 2002TC70 

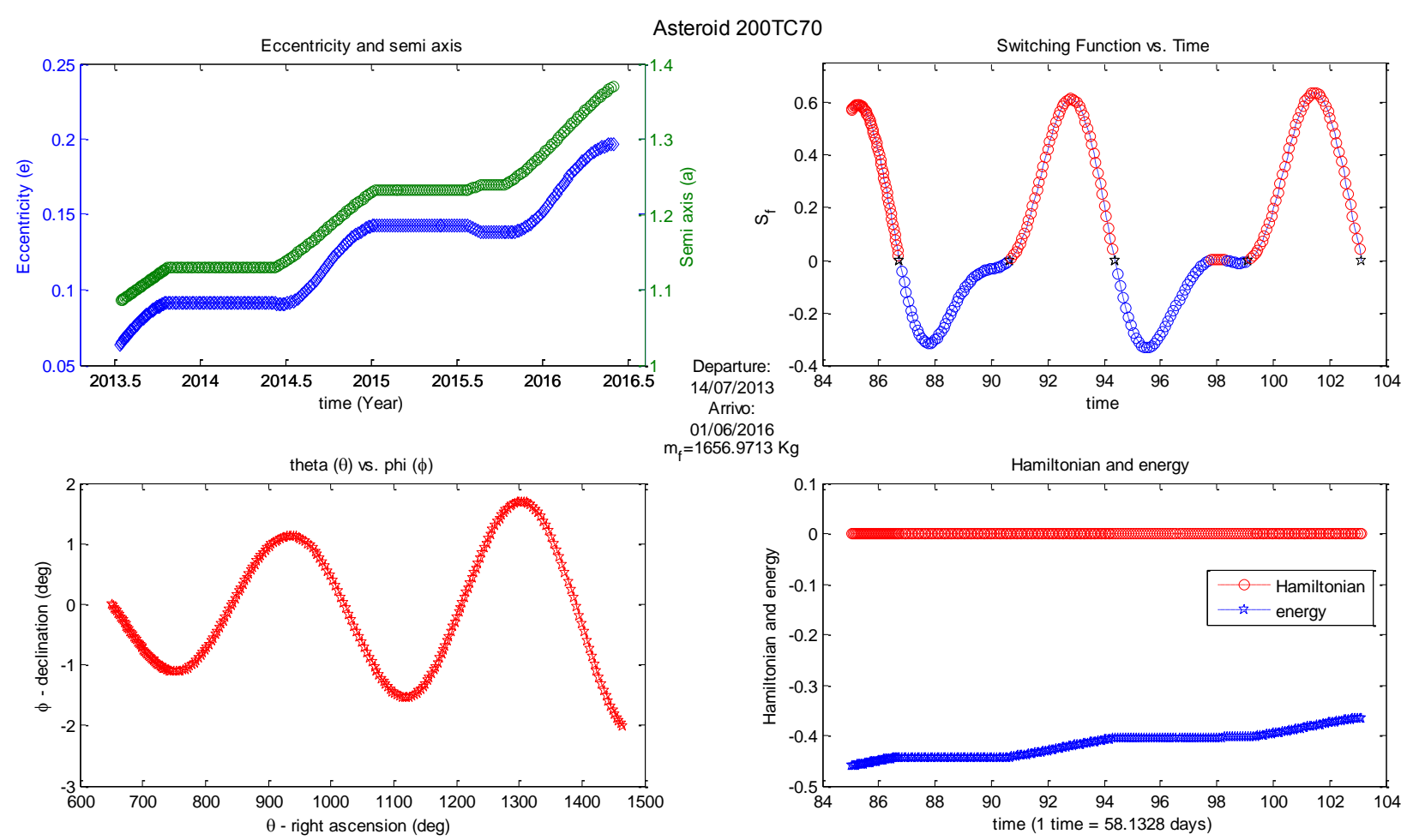

Figure 2 - Maneuver leaving from the Earth and arriving at the asteroid 2002TC.

The eccentricity (e) and semi-major axis (a); the structure of the switching function that shows the thrust arc and coast arc (Eq. 14); the right ascension as a function of the declination; the evolution of the Hamiltonian and energy orbits are visualized in Figure 2.

\subsubsection{EGA mission}

An Earth flyby can be used to vary the semi-major axis (a) and the eccentricity (e) in order to increase the apoapsis $\left(r_{a}\right)$ (or reduce the periapsis $r_{p}$ ) or to vary the inclination (i) of the orbit.

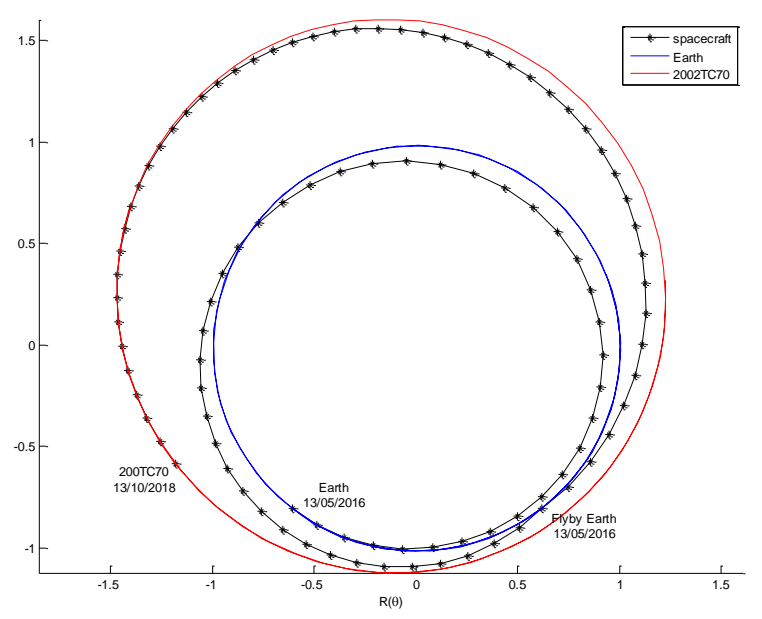

Figure 3 - Trajectory leaving the Earth and arriving to the asteroid, using EGA maneuvers.
Both the effects can be achieved if the line of nodes and the line of apsides are aligned (i.e. the argument of periapsis is close to $\omega \approx 0,180$ or 360 degrees).

\subsubsection{EMGA mission}

The formulation allows the use of multiple flyby's searching for a better performance. The criterion of the choice of flyby in Mars or Venus is the semi-major axis (a) of the asteroid:

$$
\left\{\begin{array}{l}
a>1 \rightarrow \text { Mars Flyby } \\
a<1 \rightarrow \text { Venus Flyby }
\end{array}\right.
$$

Mars flyby can be used to increase the periapsis $\left(r_{p}\right)$ and in Venus to reduce the apoapsis $\left(r_{a}\right)$.

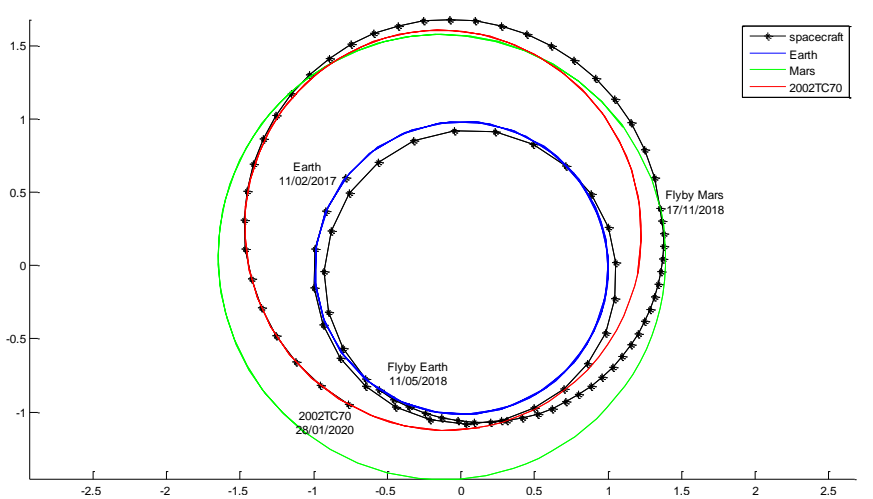

Figure $4^{-1.5}$ - Trajectory leaving the Earth and arriving to the asteroid, using EMGA maneuvers. 

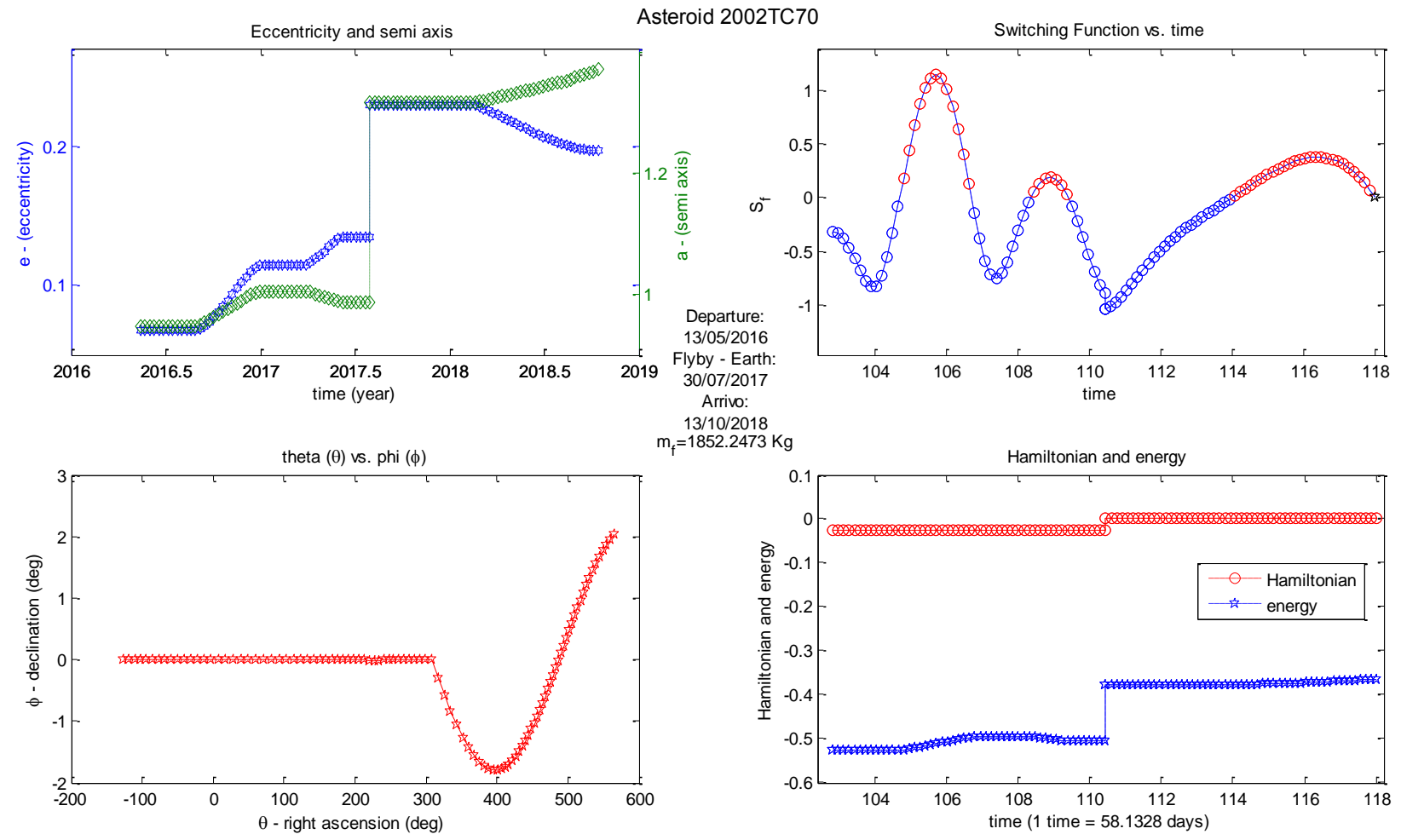

Figure 5 - Simulation with maneuver of Earth flyby to the asteroid $2002 \mathrm{TC70}$.
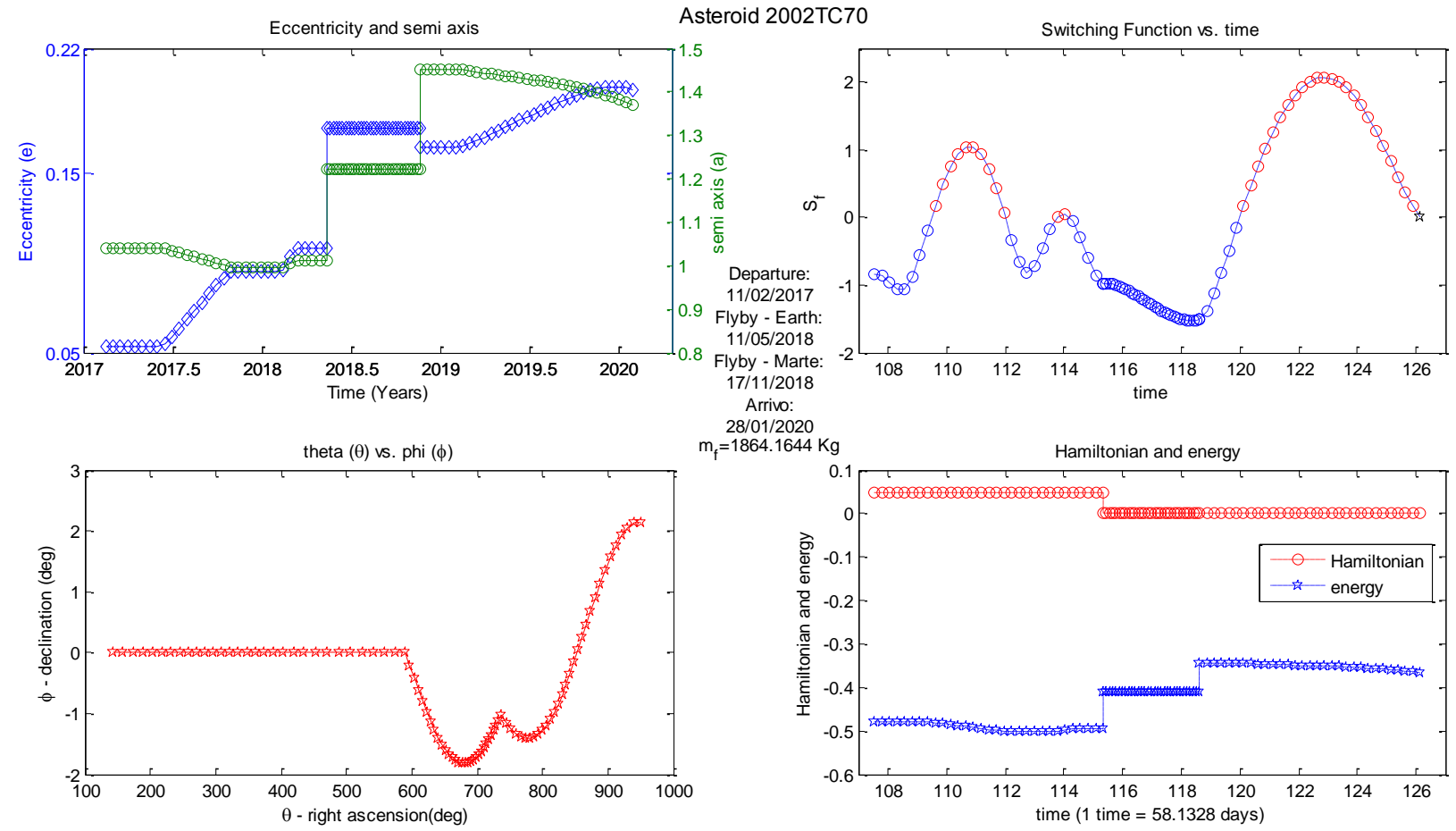

28/01/2020

$\mathrm{m}_{\mathrm{f}}=1864.1644 \mathrm{Kg}$

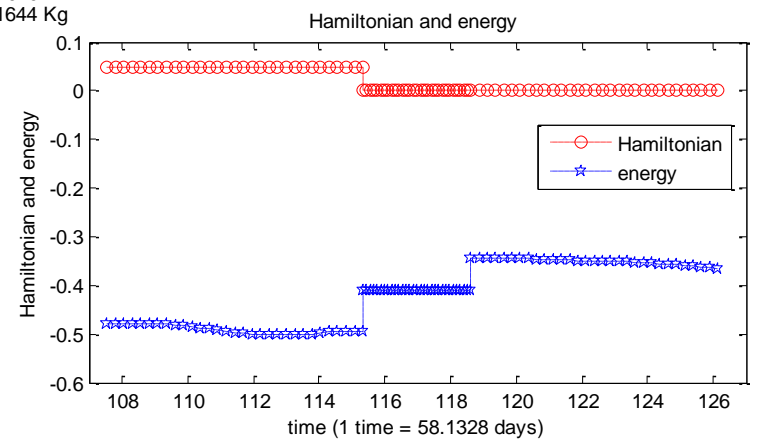

Figure 6 - Simulation with multiple flyby's: in the Earth and in Mars to the asteroid $2002 T C 70$. 
Figures 4 and 5 show the parameters of a mission leaving the Earth, making one flyby in the Earth and other in Mars, with the objective of intercepting the asteroid 2002TC70.

Figure 6 shows the transfer orbit from the Earth to asteroid 2002TC70, the switching function that shows the alternation between the propulsion arcs and the arcs without propulsion, the variation of the right ascension in comparison with the declination, the energy and the Hamiltonian of the transfer orbit.

In this section were shown a comparison of time and final mass of the spacecraft with the use of the optimized maneuver without flyby e with flyby at the Earth, and, Earth and Mars.

\subsection{NUMERICAL ANALYSIS WITH PHALL 1 (UNB)}

The researchers of the Plasma Laboratory of the Physics Institute of the Brasilia University (UNB), since 2002, pledge in the study and development of a propellant that uses a plasma propulsion system produced by current Hall, based on Stationary Plasma Thrusters (SPT). They use permanent magnets with generating the magnetic field, reducing the electricity consumption.

The characteristics of the spacecraft propulsion system are:

1. the mass of the spacecraft with an altitude of $200 \mathrm{~km}$ in circular LEO is $2133.3 \mathrm{Kg}$;

2. specific impulse $I_{\mathrm{s}}=1607 \mathrm{~s}$ :

3. specific energy $\varepsilon=0.06$;

4. $T=2 \cdot 126 \mathrm{mN}$ (thruster Phall $1-\mathrm{UNB}$ );

5. nominal thruster $\mathrm{T}_{\mathrm{O}}=1 \mathrm{UA}$;

6. The time: time $=0$ corresponds to the date 01/01/2000.

Diverse missions can be implemented with the optimization algorithm used in this work, of which the main ones are: transference with free time (to change to the space vehicle orbit without restrictions to the necessary time the execution maneuver), Gomes, [12 -13]; "Rendezvous" (one desires that the space vehicle finds and remains to the side of another space vehicle), Vaz [14]; "Flyby" (desires to intercept another celestial body, however without the objective to remain next); "Swing-By" (to pass by a celestial body to gain or to lose energy, speed and angular moment), etc.

\subsection{1 - Simulation without flyby}

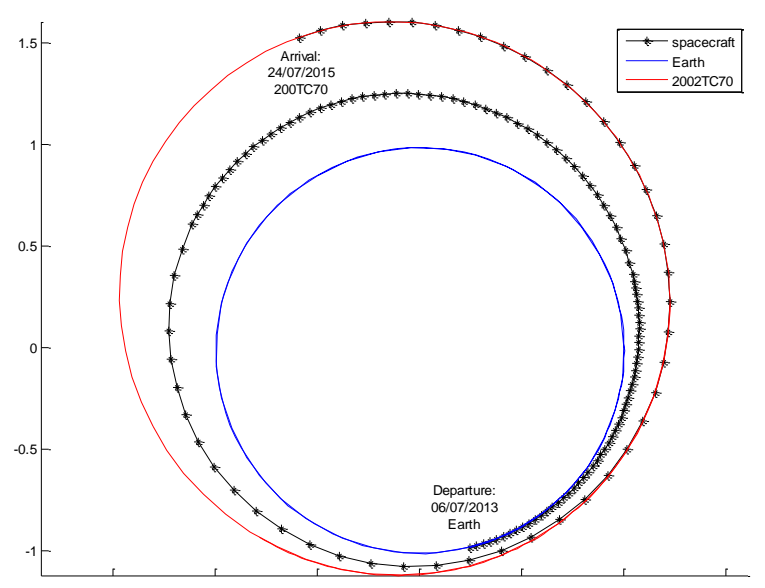

Figure 7 - Trajectory direct (without flyby) to the asteroid 2002TC70, using Phall 1.

\subsection{2 - EGA mission}

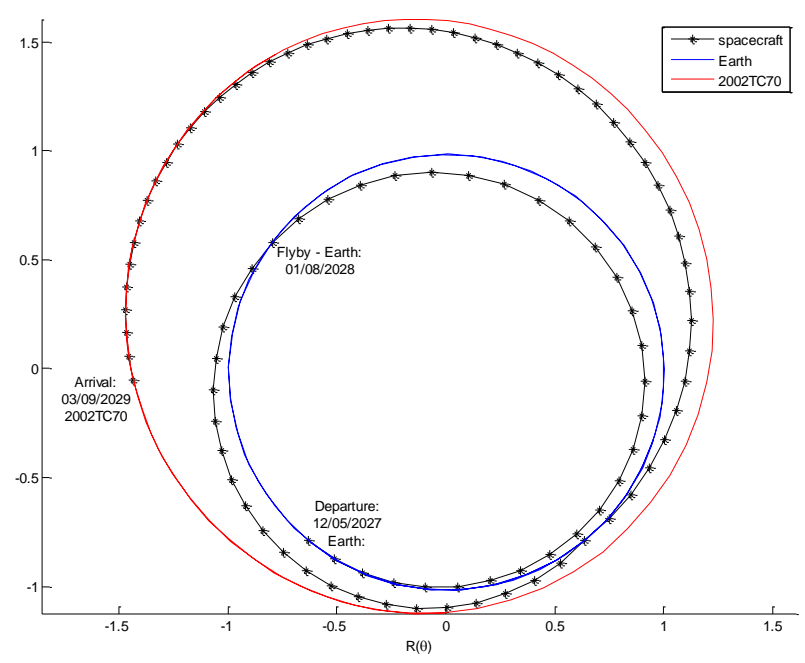

Figure 8 - Trajectory leaving the Earth and arriving to the asteroid, using EGA maneuvers, with Phall 1. 

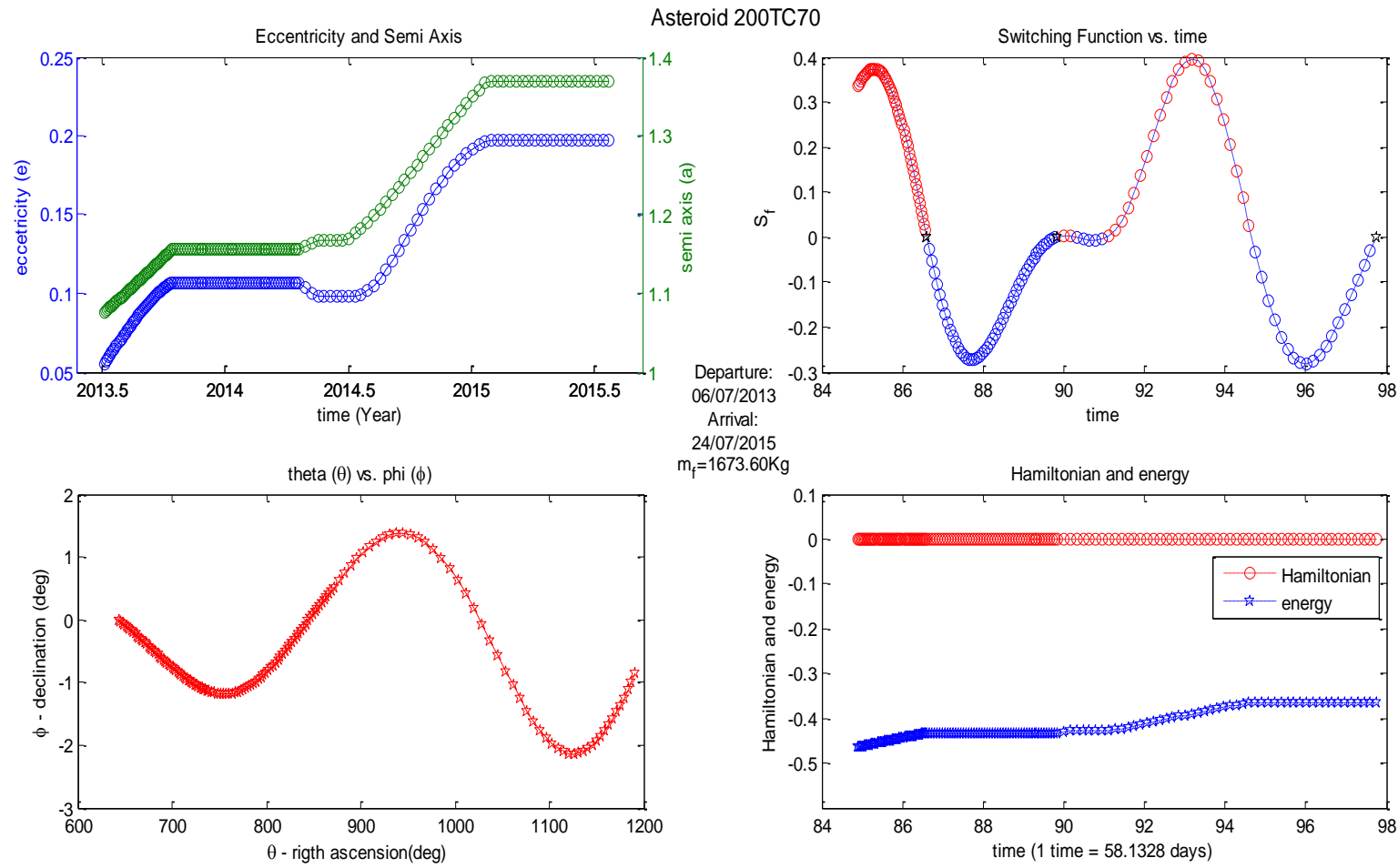

$\mathrm{m}_{\mathrm{f}}=1673.60 \mathrm{Kg}$

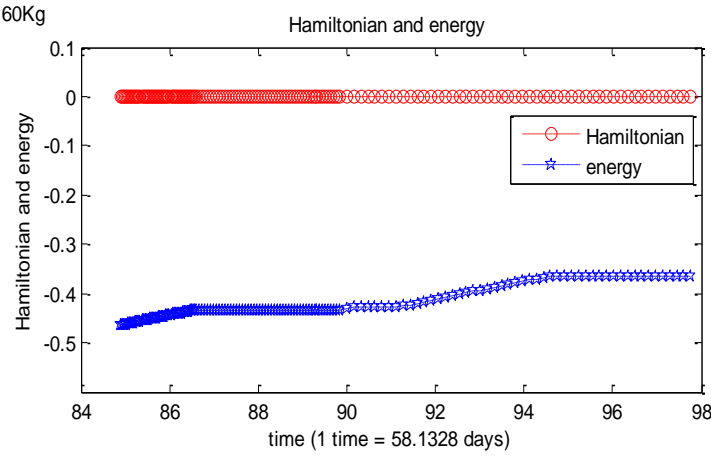

Figure 9 - Maneuver leaving from Earth and arriving to asteroid 2002TC, with Phall 1.
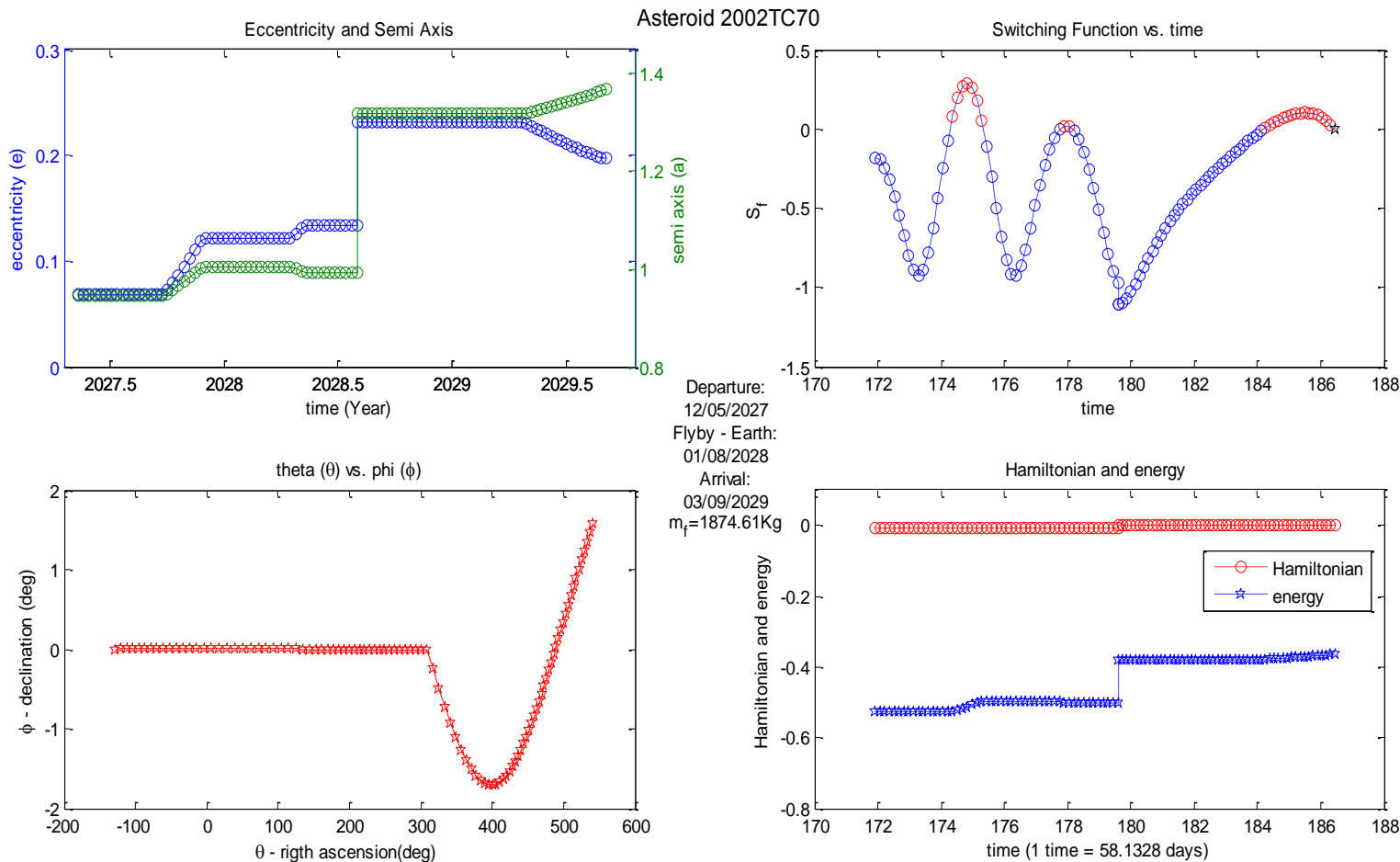

Figure 10 - Simulation with maneuver of Earth flyby to the asteroid 2002TC70, with Phall 1. 


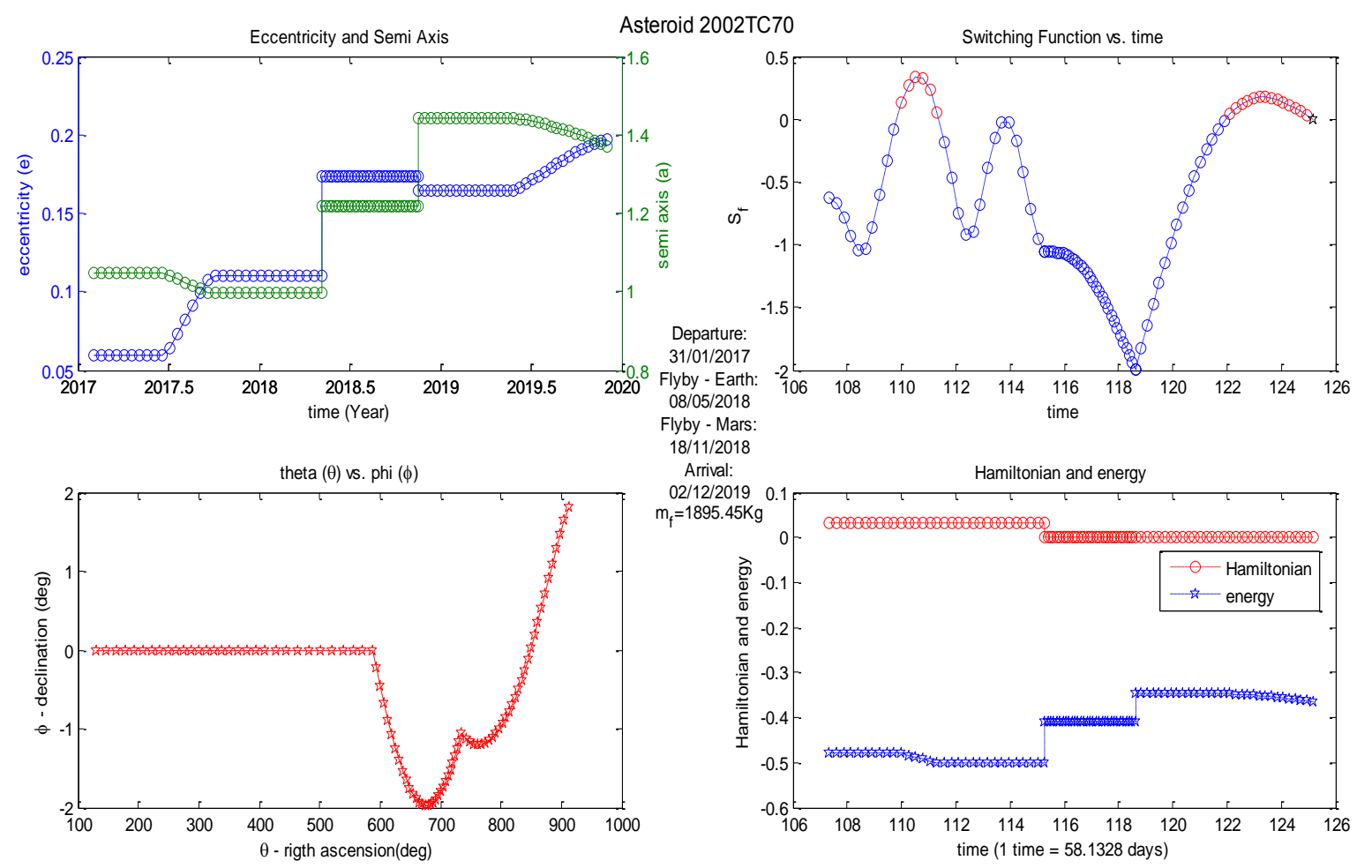

Figure 11 - Simulation with multiple flyby's: in the Earth and in Mars to the asteroid 2002TC70, with Phall 1.

\begin{tabular}{|c|c|c|c|c|c|c|c|c|c|}
\hline \multicolumn{10}{|c|}{ Asteroid 2002TC70 } \\
\hline \multicolumn{5}{|c|}{ PPS $1350\left(I_{s}=1550 s\right)(2 \times 70 \mathrm{mN})$} & \multicolumn{5}{|c|}{ Hall $($ UNB $)(I s=1607 s)(2 \times 126 \mathrm{mN})$} \\
\hline $\begin{array}{c}\text { Duration } \\
(\text { days })\end{array}$ & $m_{f}$ & Data & $\begin{array}{c}\Delta \mathrm{V}_{\mathrm{el}} \\
\text { (electric) } \\
(\mathrm{km} / \mathbf{s})\end{array}$ & $\begin{array}{c}\mathbf{V}_{\infty} \\
(\mathbf{k m} / \mathbf{s})\end{array}$ & $\begin{array}{c}\text { Duration } \\
\text { (days) }\end{array}$ & $m_{f}$ & Data & $\begin{array}{c}\Delta \mathrm{V}_{\text {el }} \\
\text { (electric) } \\
(\mathrm{km} / \mathbf{s})\end{array}$ & $\begin{array}{c}\mathbf{V}_{\infty} \\
(\mathbf{k m} / \mathbf{s})\end{array}$ \\
\hline \multicolumn{5}{|c|}{ Flyby: 0} & \multicolumn{5}{|c|}{ Flyby: 0} \\
\hline 1052.85 & 0.7768 & $\begin{array}{c}\text { Departure: } \\
\text { 14/07/2013 } \\
\text { Arrival: } \\
01 / 06 / 2016\end{array}$ & 3.83917757 & 1.25579628 & 748.24 & 0.7846 & $\begin{array}{c}\text { Departure: } \\
\text { 06/07/2013 } \\
\text { Arrival: } \\
24 / 07 / 2015\end{array}$ & 3.820898907 & 1.117814179 \\
\hline \multicolumn{5}{|c|}{ Flyby: 1 - Earth (EGA) } & \multicolumn{5}{|c|}{ Flyby: 1 - Earth (EGA) } \\
\hline 883.40 & 0.8684 & $\begin{array}{c}\text { Departure: } \\
\text { 13/05/2016 } \\
\text { Flyby - Earth: } \\
\text { 30/07/2017 } \\
\text { Arrival: } \\
\text { 13/10/2018 }\end{array}$ & 2.14480657 & 0.8376562 & 845.23 & 0.8789 & $\begin{array}{c}\text { Departure: } \\
\text { 12/05/2027 } \\
\text { Flyby - Earth: } \\
01 / 08 / 2028 \\
\text { Arrival: } \\
\text { 03/09/2029 }\end{array}$ & 2.034274163 & 0.870011798 \\
\hline \multicolumn{5}{|c|}{ Flyby: 2 - Earth - Mars (EMGA) } & \multicolumn{5}{|c|}{ Flyby: 2 - Earth - Mars (EMGA) } \\
\hline 1081.15 & 0.8739 & $\begin{array}{c}\text { Departure: } \\
\text { 11/02/2017 } \\
\text { Flyby - Earth: } \\
\text { 11/05/2018 } \\
\text { Flyby - Mars: } \\
\text { 17/11/2018 } \\
\text { Arrival: } \\
\text { 28/01/2020 } \\
\end{array}$ & 2.04883921 & 0.58081769 & 1035.51 & 0.8886 & $\begin{array}{c}\text { Departure: } \\
\text { 31/01/2017 } \\
\text { Flyby - Earth: } \\
\text { 08/05/2018 } \\
\text { Flyby - Mars: } \\
\text { 18/11/2018 } \\
\text { Arrival: } \\
\text { 02/12/2019 } \\
\end{array}$ & 1.86129921 & 0.647333385 \\
\hline
\end{tabular}

Figure 12 - Comparative table of the use of the flyby for asteroids $2002 T C 70$ using two Thrusters. 


\subsubsection{EMGA mission}

It resulted better in comparison to the results gotten with PPS 1350 (Table), therefore, Phall 1 possess a bigger specific thrust and the thruster $(\mathrm{t})$ is bigger in magnitude.

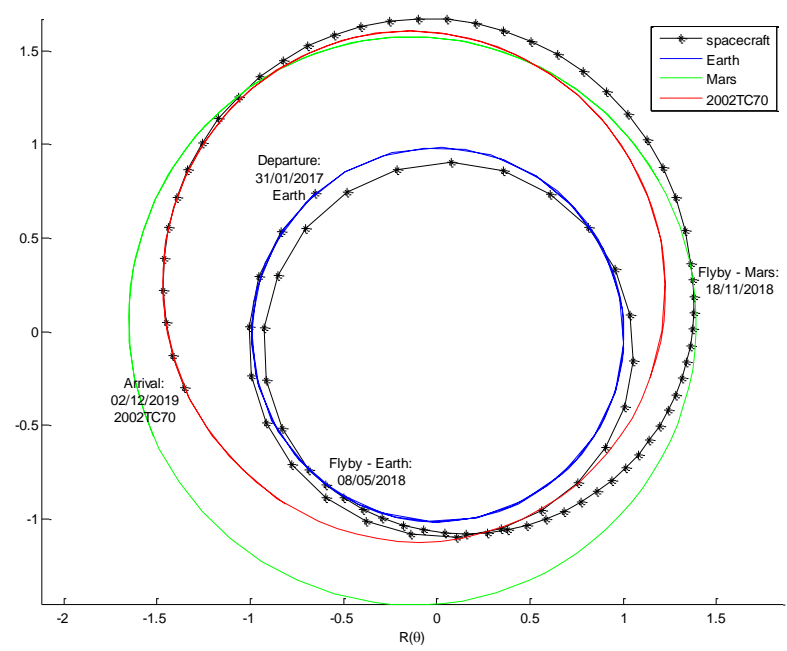

\section{Figure 13 - Trajectory leaving the Earth and arriving to the asteroid, using EMGA maneuvers, with Phall 1.}

The Figure 12 exhibit a comparison (asteroid 200TC70 using 2 thrusters: PPS 1350 and Phall) of time and final mass of the vehicle with the use of the optimized maneuver without flyby e with flyby at the Earth, Earth and Mars; also compares, the $\Delta \mathrm{V}$ electric gotten with the maneuver and the related ideal or possible dates for the mission.

Simulations with maneuvers for asteroid had been carried through 1989UQ, also using EVGA (first flyby in the Earth and second flyby in Venus) maneuvers, and the results obtained were satisfactory with the use of solar electric energy.

From numerical simulation of spacecraft orbit transfer it is possible to conclude that with higher Phall thrust will be possible to decrease maneuvering time.

\section{CONCLUSION}

The search for the best initial parameters for a mission is facilitated if the transfer orbit with free time is optimized first. The ideal asteroids for EGA missions should possess low orbit energy, perihelion close to $1 \mathrm{UA}$, low inclination per EGA.

Indirect optimization methods based on optimal control theory supply accurate solutions. The use of Gravity Assisted Maneuver (EGA, EMGA or EVGA) in this mission reduces the fuel consumption and the time of the maneuver, demonstrating that this important formulation is viable and useful.

Orbits with Phall 1 had been analyzed using gravity assisted maneuvers and verified resulted optimistical for the implantation of probes using this technology, also being able to use this formularization in the future missions that use launch vehicle that is in development/improvement (VLS-2, Brazil), which can inject in LEO (low earth orbit) a satellite medium sized, thereafter, use the solar electric propulsion (SEP) or nuclear (NEP) to dislocate the vehicle for desired orbits, maximizing them with the maneuver that use assisted gravity.

The present analysis favor a guess at the tentative solution as the Earth's positions as departure and flyby are a priori known. The ideal asteroid has perihelion radius which is close to $1 \mathrm{AU}$, a lowenergy orbit and low inclination with relation to the ecliptical axis.

The performance parameters of Phall are competitive with known electromagnet Hall thrusters found on the literature.

The fuel consumption for a mission with multiples flyby's follows the criterion of the asteroid orbit.

\section{ACKNOWLEDGMENTS}

This work was accomplished with the support of CAPES - Brazil, INPE - National Institute for Space Research - Brazil, and Instituto Politecnico de Torino - Italy.

These results are part of the work of doctoral thesis in engineering and space technologies - 
space mechanical and control, developed in INPE and Instituto Politecnico de Torino.

\section{REFERENCES}

[1]Casalino, L. and Colasurdo, G., "Missions to Asteroids Using Solar Eletric Propusion." Acta Astronautica Vol. 50, No. 11,, 2002: pp.705711.

[2]Santos, D. P. S dos., Estudo Comparativo de Diferentes Métodos de Manobras Orbitais de Empuxo Contínuo. Exame de Qualificação de Doutorado em Engenharia e Tecnologias Espaciais, São José dos Campos: INPE, 2006.

[3]Lawden, D.F., "Fundamentals of Space Navigation." JBIS, 1954: Vol. 13, pp. 87-101.

[4]Lawden, D.F., "Minimal Rocket Trajectories." ARS Journal, 1953: Vol. 23, No. 6, pp. 360382 .

[5]Casalino, L.; Colasurdo, G., and Pasttrone., "Optimal Low-Thrust Scape Trajectories Using Gravity Assist." Journal Of Guindance, Control and Dinamics,, 1999: v. 22, n. 5, p. 637-642,.

[6] Prado, A. F.B. A; Santos, D. P. S.; Rocco, E. M., "Consecutive Collision Orbit Problems Transfer Maneuvers From One Body Back To The Same Body". Icnpaa - 6th International Conference On Mathematical Problems In Engineering And Aerospace Sciences, 2006.

[7] Prado, A.F.B.A, "Low-Thrust Trajectories to the Moon", Computer and Simulation in Modern Science, Vol 1, 2008, pg 89-94. WSEAS Press, ISBN 978-960-474-010-9. Editor-in-Chief: Nikos Mastorakis.

[8] Santos, D. P. S.; Prado, A. F.B. A; Rocco, E. M., "The Use of Consecutive Collision Orbits to Obtain Swing-By Maneuvers." IAF - 56th International Astronautical Congress, 2005.

[9] Santos, D.P.S., Casalino, L, Colasurdo, G, Prado, AFBAP, "Optimal Trajectories using Gravity Assisted Maneuver and Solar
Electric Propulsion (Sep) Towards NearEarth-Objects", Proceedings of the 4th WSEAS International Conference on APPLIED and THEORETICAL MECHANICS (MECHANICS '08), pg 6268, Cairo, Egypt December 29-31, 2008.

[10] Marec, J.P., Optimal Space Trajectories. New York, NY,: Elsevier., 1979.

[11] Brophy, J. R. and Noca, M., "Eletric propulsion for solar system exploration." Journal of Propulsion and Power, 1998: 14, 700-707.

[12] Gomes, V.M., Prado, A.F.B.A, Kuga, H.K., "Orbital maneuvers Using Low Thrust", Recent Advances in Signal Processing, Robotics and Automation, pg. 120-125. WSEAS Press, ISBN 978-960-474-054-3.

[13] Gomes, V.M., Prado, A.F.B.A., "A Study of the Close Approach Between a Planet and a Cloud of Particles", Recent Advances in Signal Processing, Robotics and Automation, pg. 126-131. WSEAS Press, ISBN 978-960-474-054-3.

[14] Vaz, B.N., Prado, A.F.B.A., "Sub-Optimal Orbital Maneuvers for Artificial Satellites", WSEAS TRANSACTIONS on APPLIED and THEORETICAL MECHANICS, Issue 8, Volume 3, August 2008. 